\title{
Spectroscopic Determination of Functionalized Active Principles from Coleus aromaticus Benth Leaf Extract Using Ionic Liquids
}

\author{
Zharama M. Llarena ${ }^{1}$
}

\begin{abstract}
Green chemistry for plant extraction of active principles is the main interest of many researchers concerned with climate change. While classical organic solvents are detrimental to our environment, greener alternatives to ionic liquids are very promising for sustainable organic chemistry. This study focused on the determination of functional groups observed in the main constituents from the ionic liquid extracts of Coleus aromaticus Benth leaves using FT-IR spectroscopy. Moreover, this research aimed to determine the best ionic liquid that can separate functionalized plant constituents from the leaves of Coleus aromaticus Benth using Fourier Trans-form Infrared Spectroscopy. Coleus aromaticus Benth leaf extract in different ionic liquids, elucidated pharma-cologically important functionalized groups present in major constituents of the plant, namely, rosmarinic acid, caffeic acid and chlorogenic acid. In connection to distinctive appearance of functional groups in the spectrum and highest \% transmittance, potassium chloride-glycerol is the best ionic liquid for green extraction.
\end{abstract}

Keywords-Coleus aromaticus; ionic liquid; rosmarinic acid; caffeic acid; chlorogenic acid.

\section{INTRODUCTION}

A $\mathrm{S}$ a result of fast industrialization progress in various synthetic organic chemistry fields, academic and industrial scientists give enormous consideration on the harmful effects of chemicals and its processes on our environment. In contrast with climate change setting and growing public interest regarding global sustainability, the progressive needs for protecting our nature and developing environmental sustainability are persuading chemists to reflect how chemistry is being conducted leading to new synthetic strategies that are more atom efficient, attain mass intensification factors well, produce less waste and use less detrimental state through the execution of the twelve principles of Green Chemistry [1].

A general explanation of green chemistry is the innovation, plan and application of chemical products and its processes to lessen or to eradicate the use and production of hazardous substances. In connection with green extraction of natural products, this definition can be altered as follows: "Green extraction is based on the discovery and design of extraction processes which will reduce energy consumption, allows use

\footnotetext{
${ }^{1}$ Graduate School, Gokongwei College of Engineering, De La Salle University Manila, Philippines.
}

of alternative solvents and renewable natural products, and ensure a safe and high quality extract/product" [2].

Natural production extraction have been utilized since fire discovery. Egyptians and Phoenicians, Jews and Arabs, Indians and Chinese, Greeks and Romans, and also, Mayas and Aztecs all had pioneering extraction procedures (maceration, alembic distillation, etc.) utilized for food, perfume or medicine. Currently, all cosmetic, perfume, biofuel, food, pharmaceutical, or fine chemical industries, use extraction procedures such as maceration, decoction, infusion, percolation, pressing, steam or hydro-distillation, and soxhlet extraction. Active principles or their precursors are extracted by pharmaceutical industries by conventional methods or modern technologies [2].

Ionic liquids (ILs) have extensive potential for clean catalytic technology development. ILs can make a notable positive environmental impact as a substitute for volatile organic solvents. Volatile organic compounds are primary origin of environmental pollution [3]. These novel solvents are gaining attention as greener replacements to conventional organic solvents with the goal of promoting sustainable chemistry. Room temperature ionic liquids (RTILs), often termed as "designer solvents", have been the excellent interest of researchers in different areas since they can be adjusted for specific applications. Promising various applications of RTILs continue to increase notably because of their unique physical and chemical properties like high thermal stability, lack of inflammability, low volatility, chemical stability and great solubility with many organic compounds. Hence, these are regarded to be modern green solvents and potential substitutes to the classical volatile organic solvents [4]. ILs, neoteric solvents composed of organic cations and inorganic or organic anions, have distinct, incomparable and excellent characteristics in extracting bioactive substances [5].

Hazardous chemical replacement by more green substitutes is also an issue of present concern, in connection with green chemistry philosophy. In this setting, the green solvent introduction is a key area because solvents account for $80 \%-$ $90 \%$ of mass utilization in usual pharmaceutical/fine chemical operational process, and they are therefore accountable for majority of the waste produced by chemical industries and laboratories. Currently, most solvents used are substances derived from fossil fuels and have a detrimental impact on our health and the environment. In addition to water, biomassderived chemicals are probably the most hopeful substitutes to 
replace these detrimental solvents. Its renewable source and incomparable combination of physicochemical properties, such as high polarity, low toxicity and flammability, high boiling point, ability to form strong hydrogen bonds and to dissolve both organic and inorganic compounds (salts, acids, bases and transition metal complexes), make glycerol a good replacement to be utilized as green reaction medium for synthetic chemistry. This pioneering way to change glycerol has been very well acknowledged by the chemical society and an immense diversity of organic reactions and synthetic procedures have been successfully developed in this medium during previous years. Outstandingly, in some cases, the utilization of glycerol as solvent was observed to increases the effectiveness and selectivity of the reactions, and permitted easy product separation and catalyst recycling [6].

The medicinal value of plants to the human race is very scientifically well-established. India cultivates about 15 percent (3000 - 3500) medicinal plants, out of 20,000 medicinal plants of the world. In an estimation of 90 percent, these herbal plants are discovered growing wild in various climatic regions of the country. It is calculated that 70 to $80 \%$ of the people across the globe, rely mainly on traditional health care system and largely on herbal medicines. Nature has been an origin of medicinal plants for thousands of years and an outstanding number of new drugs have been isolated from natural sources. Various medicinal plants have been utilized for several years in daily life to mitigate different ailments worldwide. They have been utilized as natural remedies and for health care preparations. The tribal and rural people of different areas of India are highly dependent on medicinal plant therapy for treating their health care needs. Plants are rich in a variety of secondary metabolites such as tannins, terpenoids, alkaloids, flavonoids, phenols, steroids, glycosides and volatile oils [7].

This study generally aimed to determine the functional groups present in the active principles using FT-IR Spectroscopy from the leaf extract of Coleus aromaticus Benth by various ionic liquids. Furthermore, this research aimed to determine the best ionic liquid that can elucidate various functional groups present in plant constituents of Coleus aromaticus Benth leaf extract using FT-IR spectrometer.

\section{MATERIALS AND METHODS}

\section{A. Collection of Plant Material}

Fresh leaves of authenticated Coleus aromaticus Benth, free from disease, were collected from Department of Science and Technology Science Garden. The leaves were washed thoroughly 2-3 times with running water and once with distilled water. The leaves were air-dried for 3 days and dried furthermore in an oven at $60^{\circ} \mathrm{C}$ for 6 hours. The leaves of Coleus aromaticus Benth were made into powder by grinding it using mortar and pestle.

\section{B. Preparation of Ionic Liquids}

Four (4) preparations of ionic liquids were made using aluminum chloride $\left(\mathrm{AlCl}_{3}\right)$ and potassium chloride $(\mathrm{KCl})$ as their inorganic salts. These inorganic salts were dissolved in either glycerol or vegetable oil as their organic solvent-based material. RTILs, namely, aluminum chloride-vegetable oil IL, potassium chloride-vegetable oil IL, aluminum chlorideglycerol IL, and potassium chloride-glycerol IL were used for extraction of powdered leaves of Coleus aromaticus Benth. The dissolution of ionic liquids was done in 1:2 molar ratio of inorganic salts, namely, aluminum chloride and potassium chloride, and organic solvents of glycerol and vegetable oil.

\section{Extraction of Active Constituents}

Ten (10) grams of powdered Coleus aromaticus Benth leaves were macerated in $250 \mathrm{~mL}$ of four (4) different ionic liquids, namely, aluminum chloride-glycerol IL, potassium chloride-glycerol IL, aluminum chloride-vegetable oil IL, and potassium chloride-vegetable oil IL. Plant extraction was done for 24-48 hours. Vacuum filtration was done to separate the solid materials from the filtrate. Collected filtrates of aluminum chloride-vegetable oil IL, potassium chloridevegetable oil IL, aluminum chloride-glycerol IL, and potassium chloride-glycerol IL were transferred into PET bottles at room temperature for FT-IR Spectroscopy analysis.

\section{D.FT-IR Determination}

Thermo Scientific Nicolet 6700 FT-IR spectrometer was used for Infrared Spectroscopy analysis. Four (4) samples, namely, aluminum chloride-vegetable oil IL, potassium chloride-vegetable oil IL, aluminum chloride-glycerol IL, and potassium chloride-glycerol IL were subjected to Fourier Transform Infrared Spectroscopy interpretation of present functional groups for possible identification of main active constituents of Coleus aromaticus Benth. Functional groups of principal constituents were compared with obtained FT-IR spectra operated at the wave number range of $400-4000 \mathrm{~cm}^{-1}$. After each measurement the crystalline surface was washed with demineralized water and dried with a soft paper.

\section{RESULTS AND DISCUSSION}

Green extraction is a promising solution for separation of active principles from plant material without damaging our environment. Replacement of volatile organic solvents should be done in order to lessen the emission of harmful compounds that influence climate change. Green Chemistry is an innovative way of promoting eco-friendly chemicals to be used for extracting active constituents responsible for the various pharmacological actions exhibiting antioxidant, anti-fungal, anti-microbial, anti-inflammatory, and anti-tumor growth properties that can improve the health and status of every person suffering from a mild to serious complication brought about by harmful contacts with the environment.

Coleus aromaticus Benth leaf extract in aluminum chloridevegetable oil ionic liquid as illustrated in Figure 1, shows three (3) weak and sharp bands at $2853.53 \mathrm{~cm}^{-1}, 2923.63 \mathrm{~cm}^{-1}$, and $3005.51 \mathrm{~cm}^{-1}$ which indicate the presence of vibration stretching of aromatic $(\mathrm{C}=\mathrm{C})$ group, whereas, the appearance of three (3) sharp bands at $1115.66 \mathrm{~cm}^{-1}, 1162.14 \mathrm{~cm}^{-1}$, and $1236.20 \mathrm{~cm}^{-1}$ shows the presence of vibration stretching of hydroxyl (-OH) group. Furthermore, sharp and weak band at $720.76 \mathrm{~cm}^{-1}$ indicates presence of vibration of stretching of 
alkenes, while, sharp band at $1744.96 \mathrm{~cm}^{-1}$ shows the presence of vibration stretching of carbonyl $(\mathrm{C}=\mathrm{O})$ group.

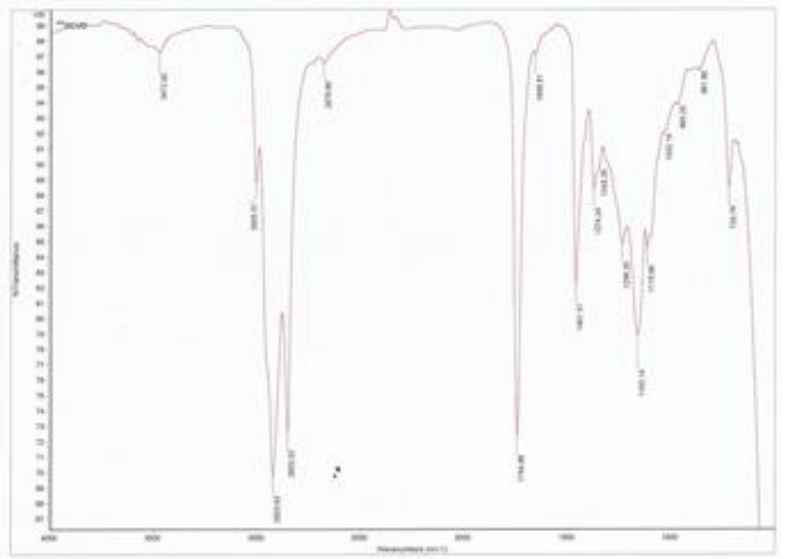

Fig. 1: Aluminum Chloride-Vegetable Oil Ionic Liquid

Leaf extract of Coleus aromaticus Benth in potassium chloride-vegetable oil ionic liquid as shown in Figure 2, illustrates same functional groups present in similar wave numbers, namely, three (3) sharp and weak peaks at 2854.06 $\mathrm{cm}^{-1}, 2924.39 \mathrm{~cm}^{-1}$, and $3005.59 \mathrm{~cm}^{-1}$ which indicate the presence of vibration stretching of aromatic $(\mathrm{C}=\mathrm{C})$ group, whereas, the appearance of three (3) sharp bands at 1116.21 $\mathrm{cm}^{-1}, 1164.10 \mathrm{~cm}^{-1}$, and $1237.12 \mathrm{~cm}^{-1}$ shows the presence of vibration stretching of hydroxyl $(-\mathrm{OH})$ group. Furthermore, weak and sharp peaks at $722.34 \mathrm{~cm}^{-1}$ indicates presence of vibration of stretching of alkenes, while, sharp peak at 1744 $\mathrm{cm}^{-1}$ shows the presence of vibration stretching of carbonyl $(\mathrm{C}=\mathrm{O})$ group. \% Transmittance $(\% \mathrm{~T})$ of functional groups is higher than $\mathrm{AlCl}_{3}$-vegetable oil ionic liquid. Hence, ionic liquid in $\mathrm{KCl}$-vegetable oil ionic liquid absorbs more active principles than Figure 1.

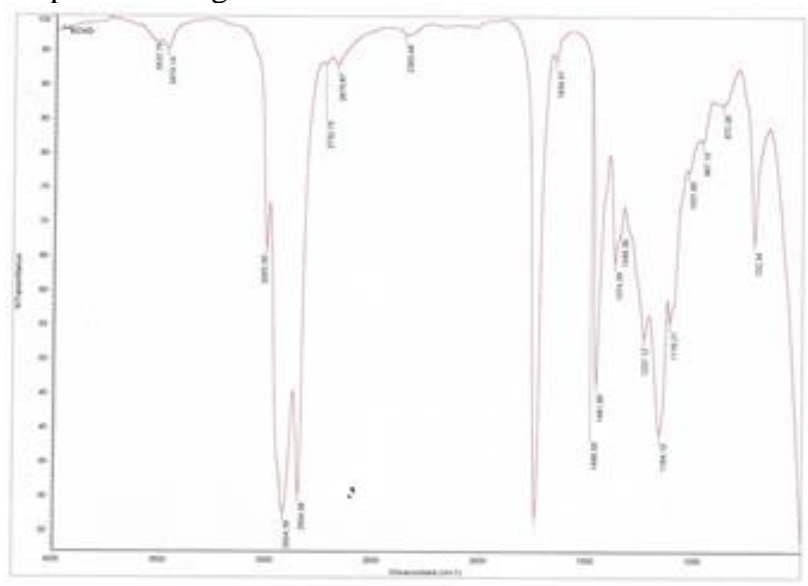

Fig. 2: Potassium Chloride-Vegetable Oil Ionic Liquid

Leaf extraction from Coleus aromaticus Benth using aluminum chloride-glycerol ionic liquid as exhibited in Figure 3 , illustrates the strong, broad band at $3374.37 \mathrm{~cm}^{-1}$ which shows the presence of vibration stretching of $(-\mathrm{OH})$ group, in connection with elucidation of weak and medium bands at $2889.19 \mathrm{~cm}^{-1}$, and $2944.50 \mathrm{~cm}^{-1}$ which indicates the presence of vibration stretching of aromatic $(\mathrm{C}=\mathrm{C})$ group. Moreover, sharp band at $1644.22 \mathrm{~cm}^{-1}$ illustrates the elucidation of vibration stretching of carbonyl $(\mathrm{C}=\mathrm{O})$ group. In addition to that, sharp and weak band at $854.98 \mathrm{~cm}^{-1}$ shows presence of vibration of stretching of alkenes

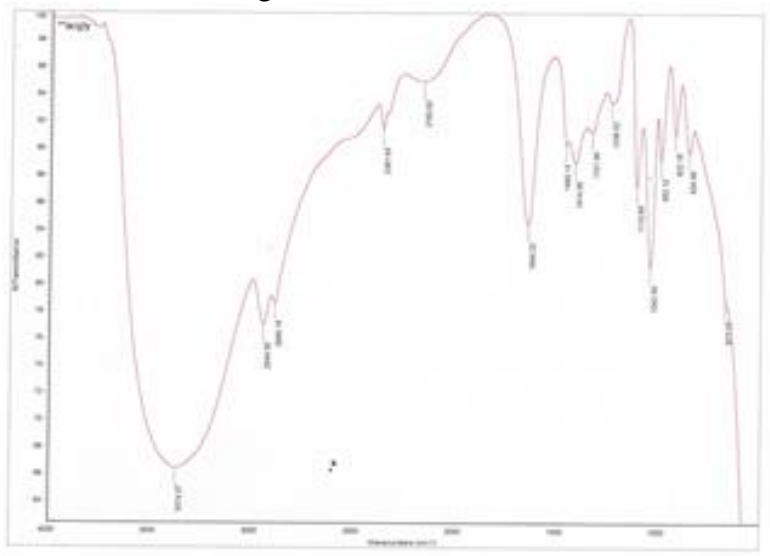

Fig. 3: Aluminum Chloride-Glycerol Ionic Liquid

Extraction of leaves from Coleus aromaticus Benth using potassium chloride-glycerol ionic liquid as illustrated in Figure 4, exhibits similar wave number of functional groups present, specifically, strong, broad peak at $3388.12 \mathrm{~cm}^{-1}$ which shows the presence of vibration stretching of hydroxyl (-OH) group, in connection with weak and medium peaks at $2888.27 \mathrm{~cm}^{-1}$, and $2944.52 \mathrm{~cm}^{-1}$ which indicate the presence of vibration stretching of aromatic $(\mathrm{C}=\mathrm{C})$ group. Moreover, sharp peak at $1644.14 \mathrm{~cm}^{-1}$ illustrates the presence of vibration stretching of carbonyl $(\mathrm{C}=\mathrm{O})$ group. In addition to that, sharp and weak peak at $852.94 \mathrm{~cm}^{-1}$ shows presence of vibration of stretching of alkenes. \% $\mathrm{T}$ of absorbed functional groups is higher than $\mathrm{AlCl}_{3}$-glycerol ionic liquid. Hence, $\mathrm{KCl}$-glycerol is the best ionic liquid for extraction of main constituents of Coleus aromaticus Benth, namely, rosmarinic acid, caffeic acid and chlorogenic acid.

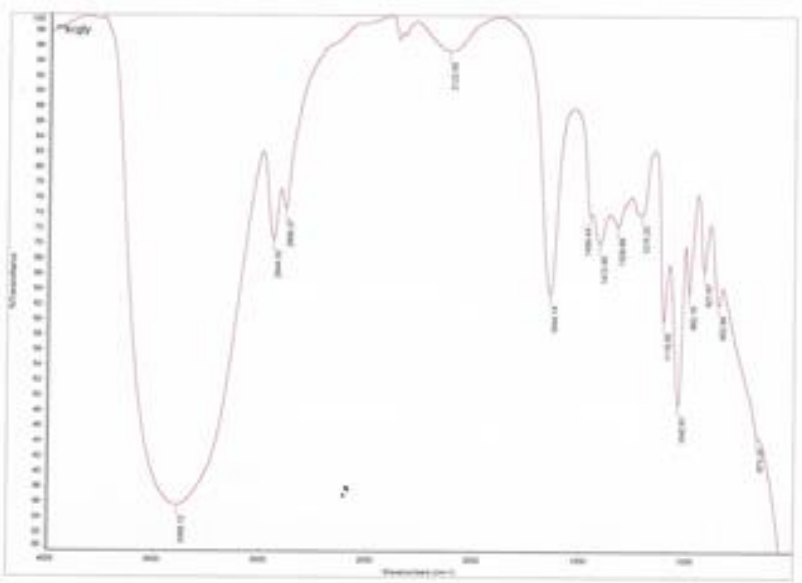

Fig. 4: Potassium Chloride-Glycerol Ionic Liquid

In connection with the shown FT-IR spectra, active principles of Coleus aromaticus Benth, namely, rosmarinic acid, caffeic acid, and chlorogenic acid have and aromatic ring and functional groups of carbonyl $(\mathrm{C}=\mathrm{O})$, hydroxyl $(-\mathrm{OH})$, and alkene $(\mathrm{C}=\mathrm{C})$ as shown in Fig. 5, 6, and 7 . 
<smiles>O=C(/C=C/c1ccc(O)c(O)c1)O[C@@H](Cc1ccc(O)c(O)c1)C(=O)O</smiles>

Fig. 5: Rosmarinic Acid

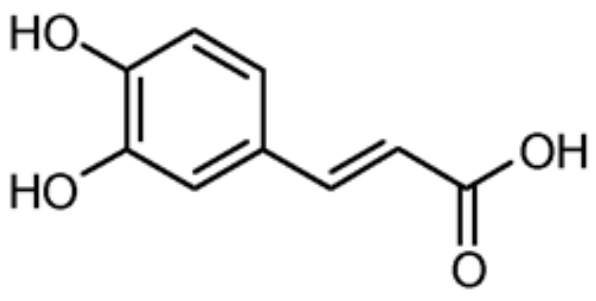

Fig. 6: Caffeic Acid

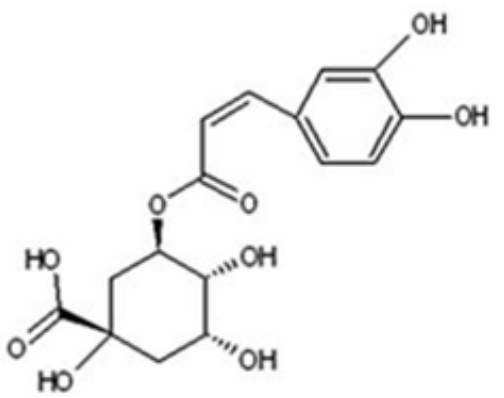

Fig. 7: Chlorogenic Acid

Phytochemicals are drugs used to treat various diseases ranging from acute to chronic infection of harmful determinants or pathogens. Plant chemicals may have same compounds present from various medicinal plants. Utilization of these herbal medicinal must be chosen from its highest possible source of active constituents. Usage of various solvents may influence the process of separating the main principles from the plant material.

From the data given, Coleus aromaticus Benth leaf extract is found to be rich in rosmarinic acid, caffeic acid and chlorogenic acid. These chief principles are found to be an effective antioxidants, anti-inflammatory and anti-cancer agents. Functional groups responsible for the antiinflammatory and anti-cancer action are the keto-groups and double bonds, whereas, $p$-hydroxyl group is accountable for the antioxidant capacity of the compound. These functional groups are readily observed in the Fourier Transform Infrared spectra. Hence, potassium chloride-glycerol IL can be used in extracting pharmacological principles leading to treatment of inflammation and cancer. These therapeutic uses are very useful in formulation of various dosage forms suitable for the utilization of ionic liquids that are affordable and environment friendly.

\section{CONCLUSION}

Green chemistry is an efficient way of replacing conventional volatile organic solvents (VOCs) that pose a serious threat, not only to our health, but also, to our environment. Designer solvents for green extraction is a better way of separating active principles from plant materials. Ionic liquids (ILs) are very promising tools of innovation for sustainable green chemistry. Room temperature ionic liquids (RTILs) show its excellent extraction process of bioactive principles without damaging our environment. Coleus aromaticus Benth leaf extract in various ionic liquids, namely, aluminum chloride-vegetable oil IL, potassium chloridevegetable oil IL, aluminum chloride-glycerol IL, and potassium chloride-glycerol IL, separated major constituents of the plant, namely, rosmarinic acid, caffeic acid and chlorogenic acid with its functional groups observed using Fourier Transform Infrared Spectroscopy, namely, $p$-hydroxyl group, double bonds and keto-group (carbonyl group). These functional groups are responsible for the treatment of cancer and inflammation, as these compounds are responsible agents for anti-inflammatory, antioxidant and anticancer. In connection to peak elucidation of functional groups in the spectrum and highest $\%$ transmittance, potassium chloride is a better inorganic salt and glycerol is a better organic solvent for separating the main constituents of Coleus aromaticus Benth. Hence, potassium chloride-glycerol is the best ionic liquid for green extraction of rosmarinic acid, caffeic acid and chlorogenic acid, illustrating the strong, broad band at 3388.12 $\mathrm{cm}^{-1}$ which exhibits the the presence of vibration stretching of hydroxyl (-OH) group, in connection with appearance of weak and medium peaks at $2888.27 \mathrm{~cm}^{-1}$, and $2944.52 \mathrm{~cm}^{-1}$ which shows the presence of vibration stretching of aromatic $(\mathrm{C}=\mathrm{C})$ group. Moreover, sharp peak at $1644.14 \mathrm{~cm}^{-1}$ indicates the presence of vibration stretching of carbonyl $(\mathrm{C}=\mathrm{O})$ group. In addition to that, sharp and weak band at $852.94 \mathrm{~cm}^{-1}$ illustrates presence of vibration of stretching of alkenes. Medicinal plants should be utilized to its utmost importance of alleviating various complications that are not in contribution of worsening the current state of our environment or will not be an added factor to climate change. Every chemical process should be done in the right way and in right balance of things in order to conform with the concepts and principles of sustainable green chemistry.

\section{V.RECOMMENDATIONS}

This study suggests of exploration of other ionic liquid extraction techniques, and observation of time kinetics needed for plant extraction. Study on other inorganic salts, other organic solvents, or other ionic liquids are also recommended. Moreover, percentage yield comparison can be further observed with other volatile organic solvents (VOCs). Furthermore, phytochemical screening may be done for chemical confirmation of active constituents. In addition to that, animal laboratory testing may also be done to study the effectiveness of the ionic liquids for extraction of bioactive compounds. Dosage formulation and its biopharmaceutical analysis may also be suggested for clinical trials using ionic liquids as a tool of extraction of main principles. 


\section{REFERENCES}

[1] Potdar, M, Kelso, G, Schwarz, L, Zhang, C, and Hearn, M, (2015): Recent Developments in Chemical Synthesis with Biocatalysts in Ionic Liquids, Molecules 2015, 20, 16788-16816 http://dx.doi.org/10.3390/molecules200916788

[2] Chemat, F, Vian, MA, and Cravotto, G, (2012): Green Extraction of Natural Products: Concept and Principles, Int. J. Mol. Sci. 2012, 13, 8615-8627 http://dx.doi.org/10.3390/ijms13078615

[3] Salminen, E, Virtanen P, and Mikkola, J, (2014): Alkaline Ionic Liquids Applied In Supported Ionic Liquid Catalyst For Selective Hydrogenation of Citral to Citronellal, Frontiers in Chemistry, February 2014, Volume 2, Article 3

[4] Ghandi, K, (2014): A Review of Ionic Liquids, Their Limits and Applications, Green and Sustainable Chemistry, 2014, 4, 44-53 http://dx.doi.org/10.4236/gsc.2014.41008

[5] Guolin, H, Jeffrey, S, Kai, Z, and Xiaolan, H, (2012): Application of Ionic Liquids in the Microwave-Assisted Extraction of Pectin from Lemon Peels, Journal of Analytical Methods in Chemistry, Volume 2012, Article ID 302059, 8 pages

[6] Diaz-Alvarez, A, and Cadierno, Victorio, (2013): Glycerol: A promising Green Solvent and Reducing Agent for Metal-Catalyzed Transfer Hydrogenation Reactions and Nanoparticles Formation, Appl. Sci., 3, 55-69 http://dx.doi.org/10.3390/app3010055

[7] Ramya, B, Ganesh, P and Kumar, R, (2012): Phytochemical Screening of Coleus aromaticus and Leucas aspera and Their Antibacterial Activity against Enteric Pathogens, International Journal of Pharmaceutical \& Biological Archives, 3(1):162-166 